\title{
Über eine Aufgabe aus der darstellenden Geometrie.*)
}

Von Wilh. Fiedler in Zürich.

Herrn Emil Waelsch's vortreffliche Behandlung einer Aufgabe aus der darstellenden Geometrie p. 92-96 bat mich an alte Überlegungen lebhaft erinnert; denn die Geraden mit zwei zusammen fallenden Orthogonalprojectionen beschlug ja meine erste Entdeckung auf darstellend geometrischem Gebiete, an welche sich der Grundgedanke meiner Reform anschloss, dass die darstellende Geometrie mit der projectivischen organisch verbunden werden müsse. Erlauben Sie, dass ich einige meiner unveröffentlichten Betrachtungen mittheile; für die veröffentlichten sehe man mein Werk "Die darstellende Geometrie in organischer Verbindung mit der Geometrie der Lage" 3. Aufl. Bd., I, §. ร3.

Die Aufgabe, die Gesammtheiten der Geraden darzustellen, von denen ein Paar Orthogonalprojectionen sich decken, ist mir ein Hauptbeispiel zur Theorie der Erzeugnisse aus der Verbindung collinearer Ebenen- und Strahlenbündel. Die projicierenden Bündel der Orthogonalprojectionen sind die Bündel der Normalebenen und Normalen zu den entsprechenden Projections- oder Coordinatenebenen, sie sind also durch die Projectionen in diesen als ihre Normalschnitte bestimmt and müssen für zwei Projectionen, welche $\mathrm{zu}$ einander congruent oder symmetrisch etc. sind, selbst gleichstimmig; resp. ungleichstimmig congruent etc. sein, natürlich auch dann, wenn jene Projectionen congruent sind und sich decken. Die Geraden unserer Aufgabe sind also die Schnittlinien der entsprechenden Ebenenpaare von solchen Bündeln und müssten bei völlig allgemeiner Lage derselben die Bisekanten-Congruenz $(1,3)$ der gewundenen Curve dritter Ordnung bilden, in welcher die Schnittpunkte entsprechender Strahlenpaare der bezüglichen Bündel liegen; der gemeinsamen Durchdringung aller der einfachen Hyperboloide und der Kegel zweiten Grades, welche aus Paaren entsprechender Ebenenbüschel beider Bündel als Orte der Schnittlinien ihrer entsprechenden Ebenen entstehen (vergl. a. a. O. Bd. III., §. 78), von lauter orthogonalen Hyperboloiden und Kegeln, weil die Paare der erzeugenden Büschel gleichwinklige sind. (a. a. O. Bd. I., §. 11,5

*) Siehe die Abhandlung von H. E. Waelsch auf p. 92 dieses Bandes.

Monatsh. f. Mathematik u. Physik. III. Jahrg. 
- die Erzeugungen aus gleichwinkligen Büscheln gehen am einfachsten aus der zugehörigen Constructionsfigur hervor; Bd. II., §. 36,12 f.; Bd. III., §. 63,2, \&. 80,5 .)

Aber die Lage unserer Bündel ist nicht allgemein, weil wir Orthogonalprojectionen combinieren, deren Ebenen auf einander oder doch auf derselben dritten Projectionsebene rechtwinklig sind; drei Projectionen auf paarweis zu einander rechtwinkligen Coordinatenebenen, wenn wir z. B. die Ebene $x z$ als Zeichnungsebene fassen und die Ebenen $x y, z y$ durch Drehung um $x$, resp. $z$ mit derselben vereinigen; drei Projectionen auf Ebenen, von denen wenigstens zwei zur dritten normal sind, wemn wir zu Grund- und Aufriss in $x y$ und $x z$ durch Einführung einer Axe $2^{x}$ einen neuen transformierten Grundriss fügen, etc. (vergl. a. a. O. Bd. I., \$. 59 f.) In jedem Falle enstpringt unsere Frage und findet im wesentlichen die gleiche Beantwortung.

Für zwei Projectionen, deren Ebenen auf einander normal sind und deren eine die Zeichnungsebene ist, sind alle Normalebenen zu ihrer Schnittlinie ( $x$ für Grund- und Aufriss, $z$ für Kreuzund Aufriss, $x$ für Aufriss und ersten Grundriss, $2^{x}$ für Aufriss und transformierten Grundriss) entsprechende vereinigte Ebenen der zugehörigen projicierenden Bündel; für zwei. Projectionen, deren Ebenen auf der Zeichnungsebene normal stehen, ist stets nur die unendlich ferne Ebene eine sich selbst entsprechende der zu den Geraden der Zeichnungsebene gehörigen projicierenden Bündel, weil die Projectionen aller unendlich fernen Geraden in der einen unendlich fernen Geraden der Zeichnungsebene vereinigt sind. Unsere Bündel sind also im ersten Falle perspectivisch oder sie projicieren das System der Geraden in einer Ebene, die durch die Schnittlinie $x, z, 2^{x}$ der letrachteten Projectionsebenen unter $45^{\circ}$ zu beiden so geht, dass auch alle ihre Punkte zusammen fallende bezügliche Projectionen haben. Im zweiten Falle aber erzeugen unsere Bündel an Stelle der gewundenen Curve dritter Ordnung einen Kegelschnitt in der unendlich fernen Ebene und eine ihn schneidende Gerade aus dem Schnittpunkte der Axen, sodass die zugehörige Bisekantencongruenz zwar wie im allgemeinen Falle durch jeden Punkt des Raumes einen Strahl schickt, aber in jeder Ebene außer der Stellung derselben, nur zwei Strahlen hat, deren Realität vom Durchschnitt der Stellung der Ebene mit jenem Kegelschnitt abhängt. (Vergl. a. a. O. Bd. III., p. 543 unten.)

Jene Gerade ist immer die Durchschnittslinie der vorher erhaltenen $45^{\circ}$ Ebenen $\mathfrak{h}_{y}$ und ibre drei Projectionen sind Punkt für Punkt vereinigt in einer Halbierungslinie des Winkels zwischen den Axen in der Zeichnungsebene. Unser Kegelschnitt $K_{\infty}$ enthält im Falle der drei in Paaren orthogonalen Projectionsebenen, auf den das Folgende beschränkt bleiben mag, außer der Richtung der Geraden $\mathfrak{h}_{y}$ die Richtungen der in der Zeichnungsebene liegenden Axen $x$ und $z$ und berüht in ihnen die Stellungen der Ebene $x y$ 
und $z y$; für den aus dem Axenschnittpunkt $O$ nach ihm gehenden Kegel ist daher $x \boldsymbol{z}$ die eine Hauptebene, die Ebenen $\mathbf{H}_{y}$ und $\mathbf{H}_{y^{\prime}}$ (a. a. O., Bd. I, \&. 46, 3 f.) sind die anderen Hauptebenen, $\mathfrak{h}$ ist die zweite Mantellinie des Hauptschnittes $\boldsymbol{H}_{y}$ und auf $\mathrm{ihm}$ sind die Tangentialebenen längs $\mathfrak{h}_{y}$ und $\mathfrak{h}$ normal. In der That decken sich für $\mathfrak{h}_{y}$ alle drei Projectionen, für $x$ und $z$ je zwei Projectionen, die die punktförmige letzte enthalten, und für hy fallen Grund- und Kreuzriss zusammen. Nach der Lage der angegebenen Elemente ist der Kegel aus gleichwinkligen Ebenenbüscheln um die Geraden $z$ und $x$ erzeugbar, für welche $z x, x y ; z \mathfrak{h}, x \mathfrak{h} ; z \mathfrak{h}_{y}, x \mathfrak{h}_{y}$ die bezüglichen Paare sind; und ebenso aus paarweis orthogonalen Ebenen um $\mathfrak{h}_{y}$ und $\mathfrak{h}$ nach den Paaren $\mathfrak{h}_{y} x, \mathfrak{h} x ; \mathfrak{h}_{y} z, \mathfrak{h} z ; \mathfrak{h}_{y} \mathfrak{h}$ and ihren Normalebenen durch $\mathfrak{h}$, resp. $\mathfrak{h}_{y}$. Seine Kreisschnitte liegen in den Normalebenen zu $\mathfrak{h}_{y}$, resp. $\mathfrak{h}$ oder in Ebenen mit gleichseitigen Spurendreiecken; seine am einfachsten darstellbaren Leitcurven sind aber die zu $\mathbf{H}_{y^{\prime}}$ parallelen elliptischen Hauptschnitte $\mathbf{S}, X Y Z_{\infty}$ mit $O X=O Y$; denn ihre ersten und dritten Projectionen $\mathbf{S}^{\prime}, \mathbf{S}^{\prime \prime \prime}$ sind die gleichen Kreise über diesen Axenabschnitten der Ebene als Durchmessern, die sich in $O$ und dem $H_{y}^{\prime, ", " ' \prime}$ der Ebene $\mathbf{S}$ oder in $\mathbf{S}, \mathfrak{h}_{y}$ orthogonal sehneiden.

Ist $H_{y}$ der Punkt von drei zusammenfallenden Projectionen für die beliebige Ebene $\mathbf{E}$ mit den Spuren $s_{1}, s_{2}, s_{3}$ oder $S_{x} S_{y}, S_{x} S_{z}, S_{z} S_{y}$, so findet man mit Benutzung des bezeichneten Hauptschnittes des Kegels $O, K_{\infty}$ die Geraden von zusammenfallendem Grund- und Kreuzriss in $\mathbf{E}$ wie folgt: Man legt die Parallelebene $\mathbf{E}^{x}$ zu $\mathbf{E}$ durch $O$ mit den Parallelen $s_{1}^{*}$ zu $s_{1}$, $s_{2}^{*} \mathrm{zu} s_{2}, s_{3}^{*} \mathrm{zu} s_{3}$ durch $O$ als Spuren und verzeichnet Grund- und Kreuzriss $d^{\prime}$, $d^{\prime \prime \prime}$ ihrer Schnittlinie $d$ mit der Hauptschnittebene $\mathbf{S}$; dann müssen ihre Schnitte mit den Kreisen $\mathbf{S}^{\prime}, \mathbf{S}^{\prime \prime}$ resp. in Paaren auf Geraden aus $O$ liegen und die Parallelen zu diesen durch $H_{y}^{\prime,}, ", \prime \prime \prime$ sind die vereinigten Grund- und Kreuzrisse der gesuchten Geraden $f_{1}, f_{2}$ der Ebene $\mathbf{E}$.

Man kann aber (vergl. a. a. O. Bd. I, §. 53, besonders pag. 282-284) diese Geraden $f_{1}^{\prime \prime}, " \prime$ und $f_{2}^{\prime \prime}, " \prime$ auch als die Doppelstrahlen der vereinigten projectivischen Strahlenbüschel bestimmen, welche von den Grund- und Kreuzrissen der Geraden durch $H_{y}$ auf $\mathbf{E}$ oder $S_{x} S_{z} S_{y}$ gebildet werden; denn man hat ein erstes Strahlenpaar $a^{\prime}, a^{\prime \prime \prime}$ derselben in den Parallelen zu $x$ und $z$ durch $H_{y}^{\prime}, "$,"', den Projectionen der zweiten Spurparallele von $\mathbf{E}_{\text {; }}$ ein zweites Paar $b^{\prime}, b^{\prime \prime \prime}$ in den Projectionen von $H_{y} S_{z}$ oder den Geraden nach $O$ und $S_{z}$; das dritte $c^{\prime}, c^{\prime \prime \prime}$ in den Projectionen von $H_{y} S_{x}$ oder den Geraden nach $S_{x}$ und $O$. Man kann für die Construction der Doppelstrablen dieser Büschel ebenso den Kreis $\mathbf{S}^{\prime}$ 
wie den Kreis $\mathbf{S}^{\prime \prime \prime}$, als Hilfskreis benutzen, da beide den Scheitel $H_{y}^{\prime}, ", " \prime$ enthalten und findet für jenen eine Pascal'sche Linie $p^{\prime}$, für diesen eine andere $p^{\prime \prime \prime}$, deren Schnitte mit $\mathbf{S}^{\prime}$ und resp. $\mathbf{S}^{\prime \prime \prime}$ in Paaren auf den beiden Geraden $f_{1}^{\prime, ' " \prime}$ und $f_{2}^{\prime, ' " \prime}$ durch $H_{y}^{\prime, ",, " \prime}$ gelegen sein müssen. Und da beide Kreise für den Mittelpunkt der Strecke $O H_{y}^{\prime,}, ", "$ zu einander centrisch symmetrisch sind, so müssen schlieblich $p^{\prime \prime}$ und $d^{\prime}$ und wieder $p^{\prime}$ and $d^{\prime \prime}$ parallel and gleich entfernt von diesem Punkte sein.

Aus $S_{x}$ und $H_{y}^{\prime, ", ", "}$ ergeben sich die Strahlenpaare $a^{\prime}, a^{\prime \prime \prime}$ und $c^{\prime}, c^{\prime \prime \prime}$ und somit ein Punkt $B$ der Pascal'schen Geraden in jedem der Hilfskreise; man kann also stets die Pascal'sche Gerade als den Durchmesser aus $B$, und man kann sie, wenn $B$ außerhalb des Hilfskreises liegt, als eine der von ihm ausgehenden Tangenten desselben wählen und somit immer eine Ebene durch $S_{x} H_{y}$ angeben, für welche die Geraden $f_{1}, f_{2}$ orthogonale Grundund Kreuzrisse haben; und in dem erwähnten Falle gibt es auch zwei Ebenen durch $S_{x} H_{y}$, für welche die Geraden mit zusammenfallenden Grund- und Kreuzrissen in einer Geraden $f_{12}^{\prime, " \prime}$ vereinigt sind. Dies geschieht auch für alle Ebenen, welche zur Grundrissund für die, welche zur Kreuzrissebene parallel sind; jenes geschieht für alle zur Aufrissebene parallelen; der Kegel $O, K_{\infty}$ schneidet jene in Parabeln mit horizontaler, resp. verticaler Axe, diese in gleichseitigen Hyperbeln mit horizontaler und verticaler Asymptote.

Wenn die Ebene $\mathbf{E}$ sich um eine feste Gerade $l$ von unbhängiger Lage dreht, so bilden ihre Geraden $f^{\prime, \prime \prime \prime}$ eine Regelfläche dritten Grades, die die Punkte von $\mathfrak{h}_{y} z u$ Doppelpunkten und die Ebenen durch $l$ zu Doppeltangentialebenen hat (vergl. a. a. O. Bd. II, §. 52,2 f.). Schneidet $l$ den Kegelschnitt $K_{\infty}$, so sondert sich das $\mathfrak{h}_{y}$ schneidende Büschel aus dieser Richtung $a b$ und die in jeder Ebene durch $l$ noch bleibende Gerade unserer Art beschreibt ein einfaches Hyperboloid, etc. So, jedoch in specieller Form, für Ebenen, die sich um eine $\mathrm{zu} x, z$ oder $\mathfrak{h}$ parallele Gerade drehen, etc.

Betrachten wir endlich die drei Projectionen desselben ebenen Systems $E$, also $E^{\prime}, E^{\prime \prime}, E^{\prime \prime \prime}$, so sind sie als affin zum System $E$ auch untereinander affin, und zwar bekanntlich $\mathbf{E}^{\prime}$ und $\mathbf{E}^{\prime \prime}$ perspectivisch affin für die Richtung von $z$ und die Affinitätsaxe $h_{x}^{\prime, "}$ oder den der Ebene zukommenden Strahl $c^{\prime}$ der vorigen Betrachtung; ferner auch E"', E" perspectivisch affin für die Richtung von $x$ und die Axe $h_{z}^{\prime \prime}, " \prime$, d. h. $H_{y} Z$ oder $b^{\prime \prime}$. Aber zwischen $E^{\prime}$ und $E^{\prime \prime}$ besteht allgemeine Affinität mit den beiden sich selbst entsprechenden oder Hauptgeraden $f_{1}^{\prime \prime \prime \prime}$ and $f_{2}^{\prime \prime \prime \prime ' . ~ D i e ~ e n t s p r e c h e n d e n ~ D r e i e c k e ~}$ $S_{x} S_{y_{1}} O$ and $O S_{y_{3}} S_{z}$ bestimmen dieselbe und dies liefert neben einer anderen Construction von $H_{y}^{\prime, ", \prime \prime \prime ~ d i e s e l b e ~ C o n s t r u c t i o n ~ d e r ~}$ Affinitätsaxen oder der $f_{i}^{\prime}, " \prime \prime$ wie oben (a. a. O. III, §. 70). 
Man erkennt das vielseitige Interesse, das die Frage des Herrn Dr. Waelsch für die Geometrie der Lage hat. Bekanntlich führt der natürliche Projectionsprocess beim Sehen des Menschen mit zwei Augen auf die allgemeine gewundene Curve dritter Ordnung als Horoptercurve und auf ihre Bisekantencongruenz $(1,3)$ : Den Ort der Punkte des Raumes, die bei der Fixierung eines bestimmten unter ihnen einfach gesehen werden und die Gesammtheit der gleichzeitig einfach gesehenen Geraden. (Siehe Helmholtz: "Handbuch der physiol. Optik“, pag. 745 f.) Das ist das schönste der hierher gehörigen Beispiele. 